\title{
3D scan line method for identifying void fabric of granular materials
}

\author{
Alexandros I. Theocharis, ${ }^{2,}$, Emmanouil Vairaktaris ${ }^{2}$, and Yannis F. Dafalias ${ }^{1,2}$ \\ ${ }^{1}$ Department of Civil and Environmental Engineering, University of California, Davis, CA, USA \\ ${ }^{2}$ Department of Mechanics, School of Applied Mathematical and Physical Science, NTUA, Athens, Attica, Greece
}

\begin{abstract}
Among other processes measuring the void phase of porous or fractured media, scan line approach is a simplified "graphical" method, mainly used in image processing related procedures. In soil mechanics, the application of scan line method is related to the soil fabric, which is important in characterizing the anisotropic mechanical response of soils. Void fabric is of particular interest, since graphical approaches are well defined experimentally and most of them can also be easily used in numerical experiments, like the scan line method. This is in contrast to the definition of fabric based on contact normal vectors that are extremely difficult to determine, especially considering physical experiments. The scan line method has been proposed by Oda et al [1] and implemented again by Ghedia and O'Sullivan [2]. A modified method based on DEM analysis instead of image measurements of fabric has been previously proposed and implemented by the authors in a 2D scheme [3-4]. In this work, a 3D extension of the modified scan line definition is presented using PFC $3 D^{\circledR}$. The results show clearly similar trends with the $2 \mathrm{D}$ case and the same behaviour of fabric anisotropy is presented.
\end{abstract}

\section{Introduction}

Soil is a multiphase granular material consisting of solid particles and voids. Among other aspects of its multiphase character, the spatial distribution of the solids and the associated voids significantly characterizes the mechanical response of the material. The technical term fabric is used to define this spatial and geometric configuration of the soil structure elements that carries a strong flavour of orientation of these elements, related to the so-called fabric anisotropy in regards to mechanical response. As Oda et al [1] and Satake [5] suggested, the most important micromechanical elements whose orientations characterize fabric anisotropy are the solid particles, the interparticle contact normal directions, and void vectors related to corresponding voids.

Void fabric is related to the position, shape and orientation of the voids, the latter defined in various ways by means of appropriate unit void vectors. In order to analytically quantify these void fabric properties, a fabric tensor that incorporates all the important parts of this information is needed, and is defined by means of the void vectors. In this work, we present a $3 \mathrm{D}$ void fabric tensor based on the scan line method initially proposed by Oda et al [1] and modified in recent works by the authors in a 2-dimensional scheme [3-4].
The basis of the scan line method is the concept that by scanning a granular material sample with parallel lines with constant distance, voids can be very easily recognized and separated from solids (Fig. 1). Thus, by using parallel lines with inclination angle between $-90^{\circ}$ and $90^{\circ}$, the orientational distribution of voids is readily recognized, for every angle.

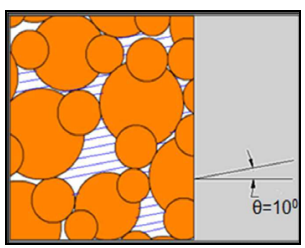

Fig. 1. Scan line 2D schematic application

Since the majority of constitutive models accounting for fabric are based on contact normal directions, and considering that contact normal fabric is more difficult to be experimentally determined than void fabric, our initial application of the modified scan line void fabric [3-4] aimed in finding a correlation between those two fabric types. This correlation has been strongly supported for 2 dimensions in [3-4]. The present work extends the applicability of that modified scan line method and explores the same correlation in 3 dimensions.

\footnotetext{
*Corresponding author: atheomec@mail.ntua.gr
} 


\section{3D fabric definitions}

The scan line fabric tensor definition uses the mean length of the void segments in each direction as a weight to quantify the void segments distribution. Extending the original 2D definition by Oda et al [1] to 3D based on spherical coordinates, one has:

$$
\mathbf{G}_{\mathrm{vs}}=\frac{1}{\sum_{\theta=-90}^{90} \sum_{\varphi=0}^{90} \ell^{\varphi, \theta}} \sum_{\theta=-90}^{90} \sum_{\varphi=0}^{90} \ell^{\varphi, \theta} \mathbf{n}_{\mathrm{vs}}^{\varphi, \theta} \otimes \mathbf{n}_{\mathrm{vs}}^{\varphi, \theta}
$$

where $\boldsymbol{n}_{\mathrm{vs}}^{\varphi, \theta}$ is the unit void vector for a point in spherical coordinates ([azimuth angle, polar angle, radial distance $]=[\theta, \varphi, 1])$ and is constant for a choice of these coordinates, $\otimes$ is the tensor product symbol, $\ell^{\varphi, \theta}$ is the mean length of scan lines (i.e. total length of line segments for angles $(\varphi, \theta)$ over the total number of scan line segments for the same angles), $\theta$ belongs to the interval $\left(-90^{\circ}, 90^{\circ}\right]$ and $\varphi$ belongs to the interval $\left(0^{\circ}, 90^{\circ}\right]$. Notice the trace of this tensor by definition is equal to 1 .

Two important parameters apply on the calculation of this tensor: (a) the distance between the lines scanning each sample and (b) the minimum length (cut off) of the scan line void segments, above which they become significant. Both these and the reason for the cut off are analysed and explained in [3].

In this 3D application, Eq. 1 is implemented along with the modification of the cut off approach; that means that the void segments whose length is less than the cut off value, are ignored in the calculation of the fabric tensor. The cut off is defined similarly as in $2 \mathrm{D}$, i.e. is equal to the minimum radius for a distribution of spheres in $3 \mathrm{D}$ and the distance between the parallel scanning lines is calibrated based on the particular sample.

The 3D contact normal fabric definition is straightforward and follows that of Satake [6]:

$$
\mathbf{G}_{\mathrm{c}}=\frac{1}{\mathrm{~N}_{\mathrm{c}}} \sum_{\mathrm{N}_{c}} \mathbf{n}_{c} \otimes \mathbf{n}_{c}
$$

where $\mathbf{n}_{c}$ is the unit contact normal vector and $\mathrm{N}_{c}$ is the number of all the contact normal vectors.

For both cases of Eqs. (1) and (2), one can take the deviatoric part of the corresponding fabric tensors as characterizing fabric with no loss of generality, a process that can relate their evolution to deviatoric plastic strain rates in continuum theories motivated by DEM results.

\section{DEM virtual experiments}

The Discrete Element Method (DEM) has been used in order to calculate the scan line void fabric tensor for a 3D specimen with spheres as solid particles. Through the DEM it is easy to apply a loading path and follow the evolution of the fabric tensors, given that all the micromechanical details are known from the numerical analysis.

We follow a typical triaxial loading path with constant mean pressure (Fig. 2a). The particles' assemblage generation was made using the radius expansion method, since this method can produce initially isotropic fabric. Also, the final stress of the equilibrium state after the radius expansion was chosen to converge to the final mean stress; in that way, the homogeneity of the sample is improved relative to other methods of particles' assemblage creation, especially for loose samples and low confining pressures.

Notice also, that loose samples are preferred from dense samples in this analysis because the later enhance non-uniform deformation and strain localisation, which complicate fabric measurements and analysis. Finally, particles have been chosen to play the role of boundaries, and the shape of the sample was decided to be spherical (Fig. 2b). The spherical shape and the boundary particles decrease the possibility of arching, and thus, of nonuniform deformation during the triaxial loading. This specimen was used in the foregoing analysis.

(a)

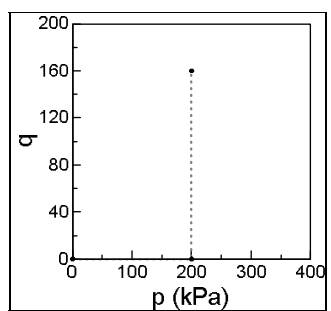

(b)

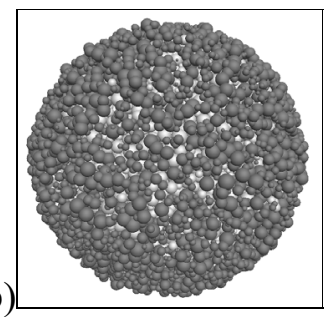

Fig. 2. (a) Loading path: isotropic compression and then triaxial compression with constant pressure $\mathrm{p}=200 \mathrm{kPa}$ and (b) sample's initial shape (dark grey are the boundary particles and light grey the sample inside)

The properties of the reference experiment are presented in Table 1. They were chosen based on the above considerations and also due to their relevance to those used in previous biaxial experiments in [3].

Table 1. Properties of the reference triaxial test.

\begin{tabular}{|l|l|}
\hline Number of grains & 13,723 \\
\hline Average grain radius $(\mathrm{m})$ & 0.001 \\
\hline $\begin{array}{l}\text { Distribution; } \\
\mathrm{r}_{\min } ; \mathrm{r}_{\max }(\mathrm{m})\end{array}$ & $\begin{array}{l}\text { Uniform; } \\
0.0005 ; 0.0015\end{array}$ \\
\hline Spherical shape initial radius $(\mathrm{m})$ & 0.0067 \\
\hline Particle Density $\left(\mathrm{kg} / \mathrm{m}^{3}\right)$ & 2600 \\
\hline Mean pressure $(\mathrm{kPa})$ & 200 \\
\hline $\mathrm{k}_{\mathrm{n}}(\mathrm{N} / \mathrm{m})$ & $2.2 \cdot 10^{4}$ \\
\hline $\mathrm{k}_{\mathrm{s}}(\mathrm{N} / \mathrm{m})$ & $2.2 \cdot 10^{4}$ \\
\hline $\begin{array}{l}\text { Surface friction } \\
\text { (ball-ball; ball-boundary ball) }\end{array}$ & $0.5 ; 0.0$ \\
\hline
\end{tabular}

\section{Fabric results}

For the triaxial loading, by applying Eq. 1 and calculating the scan line fabric tensor in 3 dimensions, the final result is a 2 nd order tensor having the nondiagonal terms very close to zero for our triaxial virtual experiment, i.e. fabric principal axes are aligned with the stress principal axes. The scan line fabric tensor 
calculations also demonstrate the symmetry of the two horizontal axes, following the equality of the two horizontal stresses. Thus, the void fabric tensor actually has two independent principal values and subsequently the fabric anisotropy intensity, directly proportional to the fabric tensor norm, can be quantified by the difference of these two principal values denoted by $\alpha_{v s}$.

The same observations apply for the contact normal fabric tensor and the respected fabric anisotropy intensity is quantified by the difference of the maximum and minimum principal values and denoted by $\alpha_{c}$. The rest of the parameters related to the application of the 3dimensional scan line method are defined and calibrated in the following paragraphs.

The distance between the scanning lines, after calibration, is set at $5 \cdot 10^{-5} \mathrm{~m}=0.00005 \mathrm{~m}$; above this limit value the results of the scan line method are meaningfully different while below this value the results do not change significantly. This limit equals $1 / 10$ of the size of the minimum grain radius.

Another important parameter of the implementation of the scan line method is the elimination of the inactive particles, i.e. particles that have less than two contacts. These particles (rattlers) in principle should be removed, as they do not contribute to the mechanical behaviour of the sample; they are thus treated as voids. On the other hand, in a real situation or physical experiment, the removal of these particles would be very difficult or impossible to account for. From that perspective two cases are examined: the first one where rattlers are treated as voids and the second one where they are treated as solids. The first case is completely compatible with the $2 \mathrm{D}$ case and is theoretically more precise, while the second one is more readily applicable.

Finally, another important issue is the volume of the scan line application. In our sample, the scan line method was applied both in the whole sample (excluding the boundaries) and inside a well-defined spherical Representative Volume (RV). The RV is representative for the macroscopic results of the sample (stress tensor, porosity); its radius is approximately $70 \%$ of the radius of the spherical sample and equal to $0.0045 \mathrm{~m}$ and it contains approximately 5000 spheres.

In the first case, the total results of the scan line apply to the whole of the inside sample (boundary particles are not considered); in the second case the results are defined, in a uniform and representative area. Both results should not vary significantly, nevertheless, they are both considered and presented in the sequel to validate the results and prove uniformity of the samples.

The previous considerations provided 4 different types of application for the scan line method in the sample:

a: scan line applied to the whole sample and no elimination of grains

b: scan line applied to the whole sample and elimination of grains with less than 2 contacts

c: scan line applied to the RV and no elimination of grains.

$\mathrm{d}$ : scan line applied to the RV and elimination of grains with less than 2 contacts

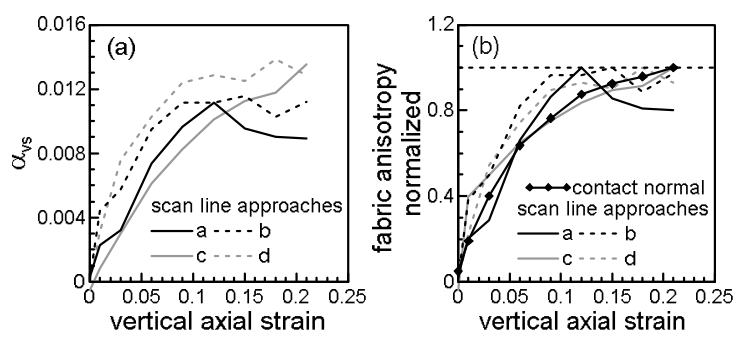

Fig. 3. Results of four variations of scan line void fabric anisotropy alongside the results for contact normal fabric anisotropy with respect to the vertical axial strain.

The evolution of the scan line anisotropy with respect to the vertical strain for all four scan line methods is presented in Fig. 3(a). The results are presented alongside the results for the contact normal fabric anisotropy in Fig. 3(b), normalized with respect to their maximum values; this kind of representation depicts the relation between the corresponding quantities with more clarity. Initially the fabric is isotropic and during the loading, anisotropy evolves gradually and monotonically - as typically for loose samples - towards its final steady state value.

The variations $\mathrm{a}$ and $\mathrm{c}$, i.e. without elimination of rattlers for the RV and for the whole sample, evolve very similarly until $12 \%$ of axial strain; then the one for the whole sample, variation a, decreases, an unexpected response for this type of specimen and loading. This happens due to the measurement details of the boundaries of the whole sample (see Fig. 2(b)). While for the RV a solid sphere is defined as the boundary for the scan line measurement, on the whole sample no definite boundary shape has been considered; the parallel scanning lines stop at the final particle they intersect, so only "limit particles" exist. This produces the difference in the void anisotropy results, especially after significant deformation of the sample ( $12 \%$ of axial strain), when the sample's shape has evolved from spherical to an elliptical one.

Additionally, Fig. 3 demonstrates that the elimination of the rattlers slightly impacts the evolution of the anisotropy (variations $b$ and $d$ versus $a$ and $c$ ). The elimination of the inactive particles produces an evolution smoother than without elimination; the intensity of the anisotropy reaches its final value in a lower axial strain and this critical state value is constant after $10 \%$ of axial strain. Notice also that variation $b$ and $\mathrm{d}$, because of these characteristics, are less qualitatively comparable to contact normal anisotropy intensity evolution, than variations a and $\mathrm{c}$.

After Figure 3, despite the inconsistency presented, it is possible to use only the first variation, a, in our analysis, i.e. scan line to the whole sample with no elimination of grains with less than 2 contacts, because it is this kind of fabric tensor that can be most easily either calculated by DEM or experimentally measured by XRay techniques, for example. Thus, we focus on the direct comparison between the scan line void fabric anisotropy and the contact normal fabric anisotropy for our reference sample. 

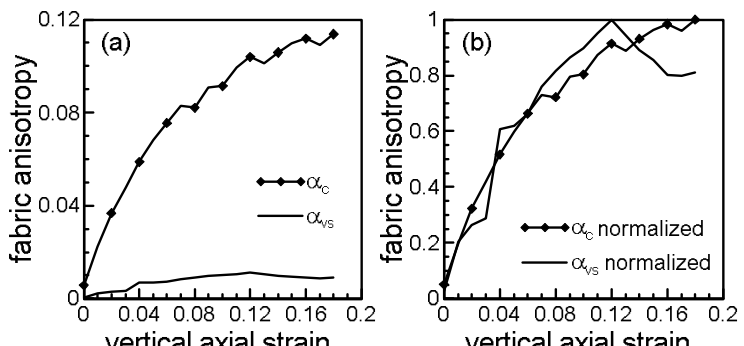

Fig. 4. Comparison between the scan line void fabric anisotropy and the contact normal fabric anisotropy (a) and their normalized values (b) with respect to the vertical axial strain.

The different scale in intensity, as presented in Fig. 4(a), is only quantitative, as the two anisotropies and the two fabric tensors are defined and calculated under a very different concept. Nevertheless, the normalized plots of anisotropies (Fig. 4(b)) show that the evolution of the two fabric anisotropies is very similar. The correlation between those two anisotropies becomes even clearer in Fig. 5. The final 5 points, that are below the correlation line, are due to the decrease of the void scan line anisotropy versus the contact normal that very slightly increases, and are due to the inconsistency discussed in the prequel.

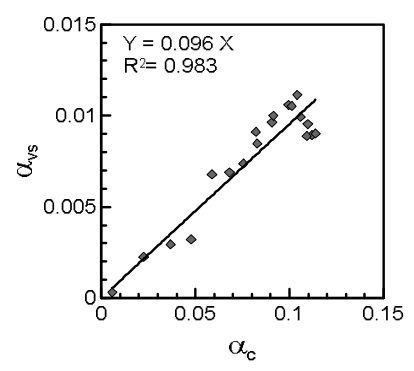

Fig. 5. Correlation between the scan line void fabric anisotropy and the contact normal fabric anisotropy

\section{Conclusions}

In this work a 3-dimensional application of the scan line method is proposed in order to quantify the void fabric of a granular medium. It is underlined that the scan line method as it has been already defined in 2D and now extended in $3 \mathrm{D}$, seems to be able to adequately quantify the void fabric anisotropy and its evolution in 3 dimensions in granular materials. In addition, a similar correlation between scan line void fabric anisotropy and the contact normal fabric anisotropy seems to exist in 3 dimensions as in 2 dimensions. The linear slope of the $3 \mathrm{D}$ correlation is 0.096 , significantly lower than the $2 \mathrm{D}$ analysis where the slope was 0.231 . This happens since the contact normal anisotropy in terms of intensity is very similar for $2 \mathrm{D}$ and $3 \mathrm{D}$, while the void scan line anisotropy intensity is in 3D one third of 2D.

The similarities between 2 and 3 dimensions are very advantageous; given that extensive research in 2 dimensions shows a clear correlation for various cases, similar behaviour can possibly be expected in 3 dimensions, towards which a more thorough investigation is in progress. Finally, a brief analysis on the importance of the inactive particles shows that the existence of those particles is not a significant barrier for the practical application of the scan line method in real cases.

The research leading to these results has received funding from the European Research Council under the European Union's Seventh Framework Program FP7-ERC-IDEAS Advanced Grant Agreement n 290963 (SOMEF). Also, Itasca Education Partnership program is acknowledged for offering access to and mentorship on the software PFC v5.0.

\section{References}

1. M. Oda, S. Nemat-Nasser, J. Konishi, Soils and Foundations, 25(3), 85-97 (1985)

2. R. Ghedia, C. O'Sullivan, Computers and Geotechnics, 41, 1-12 (2012)

3. Theocharis A., Master's thesis, National Technical University of Athens, Athens, Greece, Retrieved from https://someferc. files.wordpress.com/2017/02/2015thesis-theocharis_void_fabric_tensor.pdf(2015)

4. A. Theocharis, E. Vairaktaris, P. Fu, Y.F. Dafalias, Geomechanics from Micro to Macro, Cambridge U.K., K. Soga, K. Kumar, G. Biscontin, M. Kuo, CRC press 153 (2014)

5. M. Satake, Int. J. Eng. Sc., 30(10), 1525-1533 (1992)

6. M. Satake, Proceedings of the IUTAM conference on deformation and failure of granular materials, Delft, Balkema press, H J Luger; P A Vermeer, 63 (1982) 